\title{
Establishing the Taxa, Functional profile, and in-silico Ayurvedic Remedy of Microbiota implicated in West Nile Fever
}

\section{Preenon Bagchi ( $\nabla$ prithish.bagchi@gmail.com )}

Padmashree Institute of Management and Sciences

\section{Jemy Ratna Jovita}

Padmashree Institute of Management and Sciences

\section{Shylesh Murthy IA}

Vasishth Academy of Advanced Studies and Research (Sarvasumana Association)

\section{Article}

Keywords: West Nile fever, microbiome, Metatranscriptomics, taxonomy, functional profile, gene receptors, docking, ayurvedic medicinal herbs

Posted Date: August 3rd, 2022

DOI: https://doi.org/10.21203/rs.3.rs-1238803/v3

License: @ (i) This work is licensed under a Creative Commons Attribution 4.0 International License. Read Full License 


\section{Abstract}

West Nile fever causing microbiome is taken in this work. Culex nigripalpus mosquito is the causative factor for West Nile Virus. Using Metatranscriptomic sequencing, identified the taxa and functional profile of the microbiome is identified. Again, the receptor genes involved in West Nile fever is taken and using computer aided drug design, the novel ligands from Ayurvedic medicinal plants Ginkgo biloba, Uncaria tomentosa, Lycoris radiate and Glycyrrhiza glabra. Further, in-vitro and invivo studies can be done on the selected ligands to prove their efficiency as drugs for the disease

\section{Introduction}

West Nile fever is an infection caused by the West Nile virus, which is spread by mosquitoe, Culex nigripalpus. Culex nigripalpus is a primary vector for causing West Nile fever; they proliferate in the nutrient rich media and colonize in freshwater aquatic habitats. The microbiota of mosquitoes provides nutrition for the development and transmission of the pathogen [1]. Mosquitoes are the main vector for transmission of the virus to humans.

West Nile virus is a member of the Japanese encephalitis virus serogroup of genus Flavivirus, family Flaviviridae. It is a positive single stranded RNA virus having lipid bilayer membrane surrounded by a nucleocapsid core having length of about $11 \mathrm{~Kb}$ which is translated to single polyprotein precursor [2]. The virus is maintained in a mosquito-bird mosquito transmission cycle primarily involving Culex species mosquitoes, transmitted primarily between avian hosts and mosquito vectors. The virus replicates affecting the brain causing neuro-virulence, it turns to be neuroinvasive when it gains access to the central nervous system. The viral serine protease, NS2B-NS3 plays a crucial role in viral replication. The distribution of the virus is seen throughout Africa, the Middle East, southern Europe, western Russia, southwestern Asia, and Australia [3].

The RNA genome consisting of 5 non-coding regions, a single open reading frame coding for three viral structural proteins (capsid, membrane, envelope)and seven non-structural (NS) proteins NS1-NS2aNS2b-NS3-NS4a-NS4b-NS5, 3 non-coding regions of about 600 nucleotides. The important properties of the virus, including host range, tissue tropism, replication, assembly, and the stimulation of B and T cell immune responses. The Src family kinase c-Yes was recently reported to be important for maturation of West Nile virus particles.

Mosquito salivary components introduced at the site of infection initially target cells such as keratinocytes and dendritic cells. The virus initially replicates in dendritic cells, then spreads to regional lymph nodes and into the bloodstream and potentially to central nervous system. The expression of the central nervous system of the chemokine receptor CCR5 and its ligand CCL5 are prominently upregulated by the West Nile virus

The virus is transmitted to humans through non-vector mediated routes. the intrauterine route, through breast milk (from mother to child) blood transfusion, a bone marrow transplant and organ transplantation.

The individuals infected with this virus are asymptomatic. The incubation period is $2-14$ days before the onset of symptoms. The severity ranges from mild encephalitic disease to a more severe from characterized by coma and death. Some individuals develop flu-like symptoms i.e., fever, headache, malaise, myalgia, fatigue, skin rash, lymph adenopathy, vomiting, and diarrhoea etc. Immuno-compromised individuals develop severe neuro-invasive diseases such as West Nile meningitis, West Nile encephalitis, and acute flaccid paralysis. Individuals with West nile fever recover within days to months Whereas those with West Nile encephalitis have a poorer outcome to recover. In acute flaccid paralysis individuals develop symptoms headache, fever, malaise, gastrointestinal upset, skin rash, and some patients have neck rigidity and changes in mental status [4].

\section{Metatranscriptomic analysis using Galaxy}

Next-generation sequencing (NGS) is an advanced version of non-Sanger-based sequencing technology that offers ultrahigh throughput, scalability, and speed. Galaxy is an open source, web-based platform for next generation computational 
biomedical research [5]. Metatranscriptomics analysis enables understanding of how the microbiome responds to the environment by studying the functional analysis of genes expressed by the microbiome [6]. The genes from the Metagenomic analysis were transcribed from functional data, active metabolic pathways can be identified in our selected microbiome community [7].

\section{Computer aided drug design}

Drug design is the whole process of taking a newly discovered compound or drug molecule. Structure based drug designing technique is used here to build, display, simulate and analyze the molecular structure. Here we have used SWISS-MODEL tool [8] for modelling the proteins (gene receptors) responsible for West Nile fever CCR5, CLEC4M, IFITM2, IRF3. Selected models from homology modelling [9] output are docked with selected phytocompound from medicinal herbs. Selection of phytocompounds was done using Lipinski rule for drug which is based on the ADME properties. Molecular docking [10] was done using Patchdock tool and best interacting phytocompounds with the gene receptors can be selected as ligands.

\section{Materials And Methods}

West Nile viruses' fastq sequences SRR10017187.1.1 and SRR10017187.1.2 were retrieved from SRA database.

Sequence's quality was checked using FASTQC [11]. MultiQC [12] was done to aggregate results from FASTQC analyses into a single report.

Sequences were trimmed using cutadapt.

FASTQC followed by MultiQC was re-run using the results of cutadapt.

Next, using SortMeRNA tool [13-17] any reads identified as rRNA in dataset was removed.

Next, using FASTQ INTERLACE tool [18] paired end FASTQ reads from two separate files were joined.

MetaPhIAn tool [19] was used for profiling the composition of microbial communities (Bacteria, Archaea and Eukaryotes) from our microbiota.

Krona tool $[20,21]$ was used to visualize the results of a metagenomic profiling as a zoomable pie chart and GraPhIAn tool [22] for visualizing high-quality circular representations of taxonomic and phylogenetic trees.

Further, HUMAnN [23] pipeline was used for efficiently and accurately profiling the presence/absence and abundance of microbial pathways in our microbiota.

Next, using the genes present in our microbiota, their 3d structure was modeled using SWISS-MODEL [8].

Phyto-compounds were downloaded from PUBCHEM.

Using, molinspiration software [24], following the principles of Lipinski's rule of five, phytocompounds were selected for docking.

Further, docking was performed using patchdock [26, 27].

\section{Results And Discussion}

Metagenome, having accession number SRR10017187, for West Nile virus was downloaded from SRA database. 
As, per Per base sequence quality results of FASTQC and MultiQC, the sequence quality is not good hence we go ahead with trimming the sequence.

CUTADAPT tool [27] is used for trimming. It finds and removes adapter sequences, primers, poly-A tails, and other types of unwanted sequence from our data. It searches for the adapter in all reads and removes it when it finds it. Further, sequence quality of the cutadapt output is checked using FASTQC and MultiQC and it is found within the range.

SortMeRNA tool removes any reads identified as rRNA from our dataset. Fastq Interlace tool joins paired end FASTQ reads from two separate files. Taxonomic profiling [28] was done using MetaPhIAn tool (Fig. 1, Table 1). The output is visualized using Krona and Graphlan (Fig. 2).

Table 1

MetaPhIAn: Predicted taxon relative abundances at each taxonomic levels

\begin{tabular}{|c|c|c|c|c|c|c|c|c|c|c|c|c|}
\hline $\begin{array}{l}\text { king } \\
\text { dom }\end{array}$ & $\begin{array}{l}\text { kingd } \\
\text { om i } \\
d\end{array}$ & phylum & class & order & $\begin{array}{l}\text { famil } \\
\mathrm{y}\end{array}$ & $\begin{array}{l}\text { fami } \\
\text { ly i } \\
\text { d }\end{array}$ & $\begin{array}{l}\text { genu } \\
\mathrm{s}\end{array}$ & $\begin{array}{l}\text { gen } \\
\text { us i } \\
\text { d }\end{array}$ & species & $\begin{array}{l}\text { speci } \\
\text { es i } \\
\text { d }\end{array}$ & $\begin{array}{l}\text { str } \\
\text { ain } \\
\mathrm{s}\end{array}$ & $\begin{array}{l}\text { strai } \\
\text { ns i } \\
\text { d }\end{array}$ \\
\hline $\begin{array}{l}\text { Viru } \\
\text { ses }\end{array}$ & $\begin{array}{l}1023 \\
9\end{array}$ & & & & & $\begin{array}{l}100 . \\
0\end{array}$ & & & & & & \\
\hline $\begin{array}{l}\text { Viru } \\
\text { ses }\end{array}$ & $\begin{array}{l}1023 \\
9\end{array}$ & $\begin{array}{l}\text { Viruses } \\
\text { unclassifi } \\
\text { ed }\end{array}$ & & & & & $\begin{array}{l}100 . \\
0\end{array}$ & & & & & \\
\hline $\begin{array}{l}\text { Viru } \\
\text { ses }\end{array}$ & $\begin{array}{l}1023 \\
9\end{array}$ & $\begin{array}{l}\text { Viruses } \\
\text { unclassifi } \\
\text { ed }\end{array}$ & $\begin{array}{l}\text { Viruses } \\
\text { unclassifi } \\
\text { ed }\end{array}$ & & & & & $\begin{array}{l}100 . \\
0\end{array}$ & & & & \\
\hline $\begin{array}{l}\text { Viru } \\
\text { ses }\end{array}$ & $\begin{array}{l}1023 \\
9\end{array}$ & $\begin{array}{l}\text { Viruses } \\
\text { unclassifi } \\
\text { ed }\end{array}$ & $\begin{array}{l}\text { Viruses } \\
\text { unclassifi } \\
\text { ed }\end{array}$ & $\begin{array}{l}\text { Viruses } \\
\text { unclassifi } \\
\text { ed }\end{array}$ & & & & & 100.0 & & & \\
\hline $\begin{array}{l}\text { Viru } \\
\text { ses }\end{array}$ & $\begin{array}{l}1023 \\
9\end{array}$ & $\begin{array}{l}\text { Viruses } \\
\text { unclassifi } \\
\text { ed }\end{array}$ & $\begin{array}{l}\text { Viruses } \\
\text { unclassifi } \\
\text { ed }\end{array}$ & $\begin{array}{l}\text { Viruses } \\
\text { unclassifi } \\
\text { ed }\end{array}$ & $\begin{array}{l}\text { Flavi } \\
\text { virida } \\
\text { e }\end{array}$ & $\begin{array}{l}110 \\
50\end{array}$ & & & & $\begin{array}{l}100 . \\
0\end{array}$ & & \\
\hline $\begin{array}{l}\text { Viru } \\
\text { ses }\end{array}$ & $\begin{array}{l}1023 \\
9\end{array}$ & $\begin{array}{l}\text { Viruses } \\
\text { unclassifi } \\
\text { ed }\end{array}$ & $\begin{array}{l}\text { Viruses } \\
\text { unclassifi } \\
\text { ed }\end{array}$ & $\begin{array}{l}\text { Viruses } \\
\text { unclassifi } \\
\text { ed }\end{array}$ & $\begin{array}{l}\text { Flavi } \\
\text { virida } \\
\mathrm{e}\end{array}$ & $\begin{array}{l}110 \\
50\end{array}$ & $\begin{array}{l}\text { Flav } \\
\text { iviru } \\
\mathrm{s}\end{array}$ & $\begin{array}{l}110 \\
51\end{array}$ & & & $\begin{array}{l}10 \\
0.0\end{array}$ & \\
\hline $\begin{array}{l}\text { Viru } \\
\text { ses }\end{array}$ & $\begin{array}{l}1023 \\
9\end{array}$ & $\begin{array}{l}\text { Viruses } \\
\text { unclassifi } \\
\text { ed }\end{array}$ & $\begin{array}{l}\text { Viruses } \\
\text { unclassifi } \\
\text { ed }\end{array}$ & $\begin{array}{l}\text { Viruses } \\
\text { unclassifi } \\
\text { ed }\end{array}$ & $\begin{array}{l}\text { Flavi } \\
\text { virida } \\
\text { e }\end{array}$ & $\begin{array}{l}110 \\
50\end{array}$ & $\begin{array}{l}\text { Flav } \\
\text { iviru } \\
\mathrm{s}\end{array}$ & $\begin{array}{l}110 \\
51\end{array}$ & $\begin{array}{l}\text { West } \\
\text { Nile } \\
\text { virus }\end{array}$ & $\begin{array}{l}1108 \\
2\end{array}$ & & $\begin{array}{l}100 \\
0\end{array}$ \\
\hline
\end{tabular}

After generation of taxonomy, we move to functional information of our microbiome. Functional information of the above microbiome community [28] was done using HUMAnN pipeline (Table 2).

Table 2

Functional information of microbiome 


\begin{tabular}{|c|c|}
\hline \# Gene Family & humann_Abundance-RELAB \\
\hline UNMAPPED & 0.888769 \\
\hline UniRef90_D9MXB1 & 0.0073362 \\
\hline UniRef90_D9MXB1|unclassified & 0.0073362 \\
\hline UniRef90_UPI0001DD3770 & 0.00495637 \\
\hline UniRef90_UPI0001DD3770|unclassified & 0.00495637 \\
\hline UniRef90_A0A1B1WW31 & 0.00484378 \\
\hline UniRef90_A0A1B1WW31|unclassified & 0.00484378 \\
\hline UniRef90_B9DF73 & 0.00459488 \\
\hline UniRef90_B9DF73|unclassified & 0.00459488 \\
\hline UniRef90_A0A067ZQ74 & 0.00422115 \\
\hline UniRef90_A0A067ZQ74|unclassified & 0.00422115 \\
\hline UniRef90_A0A077EZ14 & 0.00417222 \\
\hline UniRef90_A0A077EZ14|unclassified & 0.00417222 \\
\hline UniRef90_A0A067ZS80 & 0.00390475 \\
\hline UniRef90_A0A067ZS80|unclassified & 0.00390475 \\
\hline UniRef90_UPI0003F054D7 & 0.00376104 \\
\hline UniRef90_UPI0003F054D7|unclassified & 0.00376104 \\
\hline UniRef90_F1BA45 & 0.0036982 \\
\hline UniRef90_F1BA45|unclassified & 0.0036982 \\
\hline UniRef90_A0A1X3CH90 & 0.00318336 \\
\hline UniRef90_A0A1X3CH90|unclassified & 0.00318336 \\
\hline UniRef90_UPI000D0C92C0 & 0.00302072 \\
\hline UniRef90_UPI000D0C92C0|unclassified & 0.00302072 \\
\hline UniRef90_K9L2G3 & 0.00265002 \\
\hline UniRef90_K9L2G3|unclassified & 0.00265002 \\
\hline UniRef90_A0A1B1WWN4 & 0.00258533 \\
\hline UniRef90_A0A1B1WWN4|unclassified & 0.00258533 \\
\hline UniRef90_K7ET80 & 0.00256502 \\
\hline UniRef90_K7ET80|unclassified & 0.00256502 \\
\hline UniRef90_Q91CD9 & 0.00251161 \\
\hline UniRef90_Q91CD9|unclassified & 0.00251161 \\
\hline UniRef90_UPI00018E17CF & 0.00240202 \\
\hline UniRef90_UPI00018E17CF/unclassified & 0.00240202 \\
\hline
\end{tabular}




\begin{tabular}{|c|c|}
\hline UniRef90_G7PXF9 & 0.00234066 \\
\hline UniRef90_G7PXF9|unclassified & 0.00234066 \\
\hline UniRef90_F6Z663 & 0.00218081 \\
\hline UniRef90_F6Z663|unclassified & 0.00218081 \\
\hline UniRef90_G7MFM1 & 0.00215974 \\
\hline UniRef90_G7MFM1/unclassified & 0.00215974 \\
\hline UniRef90_A0A2K6NB76 & 0.0020891 \\
\hline UniRef90_A0A2K6NB76|unclassified & 0.0020891 \\
\hline UniRef90_UPI000181CCFE & 0.00203554 \\
\hline UniRef90_UPI000181CCFE|unclassified & 0.00203554 \\
\hline UniRef90_C9WPK8 & 0.00195453 \\
\hline UniRef90_C9WPK8|unclassified & 0.00195453 \\
\hline UniRef90_P14335 & 0.00180304 \\
\hline UniRef90_P14335|unclassified & 0.00180304 \\
\hline UniRef90_F7HJ82 & 0.00180269 \\
\hline UniRef90_F7HJ82|unclassified & 0.00180269 \\
\hline UniRef90_A0A0D3MDX0 & 0.00160595 \\
\hline UniRef90_A0A0D3MDX0|unclassified & 0.00160595 \\
\hline UniRef90_UPI0000E69E2F & 0.00153697 \\
\hline UniRef90_UPI0000E69E2F/unclassified & 0.00153697 \\
\hline UniRef90_I3VPR6 & 0.00149368 \\
\hline UniRef90_I3VPR6|unclassified & 0.00149368 \\
\hline UniRef90_G8E0A9 & 0.00146336 \\
\hline UniRef90_G8E0A9|unclassified & 0.00146336 \\
\hline UniRef90_D3X888 & 0.0013421 \\
\hline UniRef90_D3X888|unclassified & 0.0013421 \\
\hline UniRef90_UPI00003BECBC & 0.00130469 \\
\hline UniRef90_UPI00003BECBC|unclassified & 0.00130469 \\
\hline UniRef90_UPI0002C39258 & 0.00127138 \\
\hline UniRef90_UPI0002C39258|unclassified & 0.00127138 \\
\hline UniRef90_Q6VXX6 & 0.00120319 \\
\hline UniRef90_Q6VXX6|unclassified & 0.00120319 \\
\hline UniRef90_D3X8F1 & 0.0011535 \\
\hline UniRef90_D3X8F1|unclassified & 0.0011535 \\
\hline
\end{tabular}




\begin{tabular}{|c|c|}
\hline UniRef90_A0A1S6WNB4 & 0.00110115 \\
\hline UniRef90_A0A1S6WNB4|unclassified & 0.00110115 \\
\hline UniRef90_D3X8F7 & 0.00102768 \\
\hline UniRef90_D3X8F7|unclassified & 0.00102768 \\
\hline UniRef90_D3X8D8 & 0.000998791 \\
\hline UniRef90_D3X8D8|unclassified & 0.000998791 \\
\hline UniRef90_Q91KP3 & 0.000989086 \\
\hline UniRef90_Q91KP3|unclassified & 0.000989086 \\
\hline UniRef90_D9IFF5 & 0.000952321 \\
\hline UniRef90_D9IFF5|unclassified & 0.000952321 \\
\hline UniRef90_F7EY45 & 0.000912381 \\
\hline UniRef90_F7EY45|unclassified & 0.000912381 \\
\hline UniRef90_D5K1B9 & 0.00090469 \\
\hline UniRef90_D5K1B9|unclassified & 0.00090469 \\
\hline UniRef90_Q67428 & 0.000861045 \\
\hline UniRef90_Q67428|unclassified & 0.000861045 \\
\hline UniRef90_Q91AB0 & 0.000854561 \\
\hline UniRef90_Q91AB0|unclassified & 0.000854561 \\
\hline UniRef90_D3X875 & 0.000850089 \\
\hline UniRef90_D3X875|unclassified & 0.000850089 \\
\hline UniRef90_A0A2K5WQL4 & 0.000795493 \\
\hline UniRef90_A0A2K5WQL4|unclassified & 0.000795493 \\
\hline UniRef90_B1P6F1 & 0.000689513 \\
\hline UniRef90_B1P6F1|unclassified & 0.000689513 \\
\hline UniRef90_P29984 & 0.000618302 \\
\hline UniRef90_P29984|unclassified & 0.000618302 \\
\hline UniRef90_A0A0B5H4C4 & 0.000582165 \\
\hline UniRef90_A0A0B5H4C4|unclassified & 0.000582165 \\
\hline UniRef90_Q6YFU6 & 0.00051542 \\
\hline UniRef90_Q6YFU6|unclassified & 0.00051542 \\
\hline UniRef90_A0A0P0RST2 & 0.000505187 \\
\hline UniRef90_A0A0P0RST2|unclassified & 0.000505187 \\
\hline UniRef90_A0A0D5CWD5 & 0.000489091 \\
\hline UniRef90_A0A0D5CWD5|unclassified & 0.000489091 \\
\hline
\end{tabular}

Page $7 / 28$ 


\begin{tabular}{|c|c|}
\hline UniRef90_A0A068AXW2 & 0.000463204 \\
\hline UniRef90_A0A068AXW2|unclassified & 0.000463204 \\
\hline UniRef90_A0A1X3CWX3 & 0.000451315 \\
\hline UniRef90_A0A1X3CWX3|unclassified & 0.000451315 \\
\hline UniRef90_I1TEC3 & 0.000443267 \\
\hline UniRef90_I1TEC3|unclassified & 0.000443267 \\
\hline UniRef90_A0A229QXW6 & 0.000390111 \\
\hline UniRef90_A0A229QXW6|unclassified & 0.000390111 \\
\hline UniRef90_UPI0000485C0B & 0.000373856 \\
\hline UniRef90_UPI0000485C0B|unclassified & 0.000373856 \\
\hline UniRef90_Q6VXX7 & 0.000367549 \\
\hline UniRef90_Q6VXX7|unclassified & 0.000367549 \\
\hline UniRef90_H2NBX3 & 0.000356109 \\
\hline UniRef90_H2NBX3|unclassified & 0.000356109 \\
\hline UniRef90_UPI000642CDD8 & 0.000349761 \\
\hline UniRef90_UPI000642CDD8|unclassified & 0.000349761 \\
\hline UniRef90_I3N2P9 & 0.000333919 \\
\hline UniRef90_I3N2P9|unclassified & 0.000333919 \\
\hline UniRef90_D9IFG5 & 0.000299914 \\
\hline UniRef90_D9IFG5|unclassified & 0.000299914 \\
\hline UniRef90_D3X874 & 0.000283626 \\
\hline UniRef90_D3X874|unclassified & 0.000283626 \\
\hline UniRef90_Q9DP92 & 0.000282183 \\
\hline UniRef90_Q9DP92|unclassified & 0.000282183 \\
\hline UniRef90_D3X8B2 & 0.000279068 \\
\hline UniRef90_D3X8B2|unclassified & 0.000279068 \\
\hline UniRef90_A0A075FC35 & 0.000211602 \\
\hline UniRef90_A0A075FC35|unclassified & 0.000211602 \\
\hline UniRef90_B7U2U9 & 0.000200363 \\
\hline UniRef90_B7U2U9|unclassified & 0.000200363 \\
\hline UniRef90_C9WPM0 & 0.000194638 \\
\hline UniRef90_C9WPM0|unclassified & 0.000194638 \\
\hline UniRef90_A0A0P7UTC2 & 0.000191691 \\
\hline UniRef90_A0A0P7UTC2|unclassified & 0.000191691 \\
\hline
\end{tabular}




\begin{tabular}{|c|c|}
\hline UniRef90_F7FBV8 & 0.000191691 \\
\hline UniRef90_F7FBV8|unclassified & 0.000191691 \\
\hline UniRef90_G3SQL6 & 0.000191691 \\
\hline UniRef90_G3SQL6|unclassified & 0.000191691 \\
\hline UniRef90_H3BYH5 & 0.000191691 \\
\hline UniRef90_H3BYH5|unclassified & 0.000191691 \\
\hline UniRef90_P60866 & 0.000191691 \\
\hline UniRef90_P60866|unclassified & 0.000191691 \\
\hline UniRef90_D3X8C7 & 0.000186455 \\
\hline UniRef90_D3X8C7|unclassified & 0.000186455 \\
\hline UniRef90_A0A2K6EGR5 & 0.000170037 \\
\hline UniRef90_A0A2K6EGR5|unclassified & 0.000170037 \\
\hline UniRef90_A0A0B7JKQ7 & 0.000166418 \\
\hline UniRef90_A0A0B7JKQ7|unclassified & 0.000166418 \\
\hline UniRef90_UPI0005215E94 & 0.000165638 \\
\hline UniRef90_UPI0005215E94|unclassified & 0.000165638 \\
\hline UniRef90_G3I2D3 & 0.00016095 \\
\hline UniRef90_G3I2D3|unclassified & 0.00016095 \\
\hline UniRef90_A0A2I0MVY6 & 0.000152329 \\
\hline UniRef90_A0A2IOMVY6|unclassified & 0.000152329 \\
\hline UniRef90_L9LBB7 & 0.000143369 \\
\hline UniRef90_L9LBB7|unclassified & 0.000143369 \\
\hline UniRef90_UPI0006B0C551 & 0.000133289 \\
\hline UniRef90_UPI0006B0C551|unclassified & 0.000133289 \\
\hline UniRef90_A0A091DCD6 & 0.000130236 \\
\hline UniRef90_A0A091DCD6|unclassified & 0.000130236 \\
\hline UniRef90_UPI000D3231B4 & 0.00012363 \\
\hline UniRef90_UPI000D3231B4|unclassified & 0.00012363 \\
\hline UniRef90_UPI000359548B & 0.000119308 \\
\hline UniRef90_UPI000359548B|unclassified & 0.000119308 \\
\hline UniRef90_UPI00051F13F9 & 0.000116856 \\
\hline UniRef90_UPI00051F13F9|unclassified & 0.000116856 \\
\hline UniRef90_UPI0005216B30 & 0.000112244 \\
\hline UniRef90_UPI0005216B30|unclassified & 0.000112244 \\
\hline
\end{tabular}

Page 9/28 


\begin{tabular}{|ll|}
\hline UniRef90_K7P5A3 & 0.000108977 \\
\hline UniRef90_K7P5A3|unclassified & 0.000108977 \\
\hline UniRef90_L5JTI1 & $8.00319 \mathrm{e}-05$ \\
\hline UniRef90_L5JTI1|unclassified & $8.00319 \mathrm{e}-05$ \\
\hline UniRef90_Q6VYH3|unclassified & $7.08503 \mathrm{e}-05$ \\
\hline UniRef90_A0A2U3XJL7 & $7.08503 \mathrm{e}-05$ \\
\hline UniRef90_A0A2U3XJL7|unclassified & $5.7032 \mathrm{e}-05$ \\
\hline
\end{tabular}

Next, from the gene family information, we obtain the functional information of our microbiome using Superfamily server. The Functional information of $1^{\text {st }}$ five families from Normalized gene families as detected by Superfamily (HMM library and genome assignments server) is given below (Fig. 3).

As per the information obtained from Superfamily database it is seen that the Domain D9MXB1 is seen in the region 296430 and 3-284 of the superfamily P-loop containing nucleoside triphosphate hydrolases and in the Family of RNA helicase.

A0A1B1WW31 domain, Region 1 at position 291-590, and of the superfamily Viral glycoprotein, central and dimerisation domains of Family Viral glycoprotein, central and dimerisation domains, 2 Region 2 at position 1990-2124 corrosponds to Superfamily P-loop containing nucleoside triphosphate hydrolases and Family RNA helicase and Region 3's position 582696 Superfamily E set domains with Family of Class II viral fusion proteins C-terminal domain.

B9DF73 Domain is included in Region 1 at position 670-996 and 1024-1247 of the Superfamily DNA/RNA polymerases Family RNA-dependent RNA-polymerase. 2nd Region at postion 370-621 of the Superfamily S-adenosyl-L-methioninedependent methyltransferases and Family mRNA cap methylase.

A0A067ZQ74 Domain's 1st Region at 2831-3157, 3185-3408 of Superfamily DNA/RNA polymerases of Family RNAdependent RNA-polymerase. $2^{\text {nd }}$ Region at 291-590 of Superfamily Viral glycoprotein, central and dimerisation domains of Family Viral glycoprotein, central and dimerisation. 3rd Region at 1990-2124 of Superfamily P-loop containing nucleoside triphosphate hydrolases in the Family RNA helicase. 4th Region at 582-696 of Superfamily E set domains of Family Class II viral fusion proteins C-terminal domain. $5^{\text {th }}$ Region at 1687-1978 of Superfamily P-loop containing nucleoside triphosphate hydrolases of the Family RNA helicase. 6th Region at 1510-1676 of the Superfamily Trypsin-like serine proteases of Family Viral proteases. 7th Region at 22-99 of Superfamily Flavivirus capsid protein C of Family Flavivirus capsid protein C and 8th Region of Superfamily S-adenosyl-L-methionine-dependent methyltransferases of FamilymRNA cap methylase.

A0A077EZ14 Domain's 1 Region at 485-619 of the Superfamily P-loop containing nucleoside triphosphate hydrolases of Family RNA helicase. Region 2 at 182-473 of Superfamily P-loop containing nucleoside triphosphate hydrolases of Family RNA helicase and Region 3 at 5-171 of Superfamily Trypsin-like serine proteases of Family Viral proteases.

\section{Structure based drug designing of West Nile Fever}

Since, West Nile feveris a mosquito-borne disease, we further go ahead towards designing novel drug for the disease. From the MetaPhIAn: Bowtie2 output we get the gene ids. Corresponding gene receptors are taken from NCBI for our work (Table 3).

Table 3

Genes with their NCBI Accession number 


\begin{tabular}{|llll|}
\hline SI. No & Gene Receptors & NCBI Accession Number & Homologous Template \\
\hline 1. & CCR5 & AAB57793.1 & 4MBS \\
\hline 2 & CLEC4M & AAI10615.1 & 1SL6 \\
\hline 3 & IFITM2 & CAG33175.1 & $3 W X X$ \\
\hline 4 & IRF3 & AAH71721.1 & 7JFM \\
\hline
\end{tabular}

\section{Abbreviations of genes:}

1. CCR5: C-C Motif Chemokine Receptor 5

2. CLEC4M: C-Type Lectin Domain Family 4 Member M

3. IFITM2: Interferon Induced Transmembrane Protein 2

4. IRF3: Interferon Regulatory Factor 3

\section{Homology modeling}

Homology modeling of the above receptors are done using SWISS-MODEL server. The receptor model and corresponding ramachandran plot results are given in Fig. 4. Template used for modeling is given in Table 3.

Ayurvedic Medicinal plants Ginkgo biloba, Uncaria tomentosa, Lycoris radiate and Glycyrrhiza glabra are traditionally used to treat many diseases, such as respiratory disorders and fever. The potency of their phytocompounds in treating West Nile Fever is studied here.

As per Lipinski's rule of five [ADME(Adsorption, distribution and metabolism extraction)] we check the drug likeliness of the above phytocompounds (Table 4-7).

Table 4

ADME studies of Ginkgo biloba 


\begin{tabular}{|c|c|c|c|c|c|c|c|c|c|c|}
\hline $\begin{array}{l}\text { SL. } \\
\text { NO }\end{array}$ & Compounds & $\begin{array}{l}\text { Mi } \\
\text { Log } \\
\text { P }\end{array}$ & TSPA & Natoms & mw & nON & nOHNH & $\begin{array}{l}\mathrm{N}- \\
\text { Violations }\end{array}$ & nROTB & VOLUME \\
\hline 1 & Ginkgolide A & -1.46 & 128.60 & 29 & 408.40 & 9 & 2 & 0 & 1 & 339.84 \\
\hline 2 & Ginkgolide B & -2.38 & 148.83 & 30 & 424.40 & 10 & 3 & 0 & 1 & 347.88 \\
\hline 3 & Ginkgolide C & -3.29 & 169.06 & 31 & 440.40 & 11 & 4 & 1 & 1 & 355.93 \\
\hline 4 & Isorhamnetin & 1.99 & 120.36 & 23 & 316.26 & 7 & 4 & 0 & 2 & 257.61 \\
\hline 5 & Quercetin & 1.68 & 131.35 & 22 & 302.24 & 7 & 5 & 0 & 1 & 240.08 \\
\hline 6 & $\begin{array}{l}\text { Protocatechuic } \\
\text { acid. }\end{array}$ & 2.10 & 118.21 & 20 & 276.24 & 6 & 5 & 0 & 3 & 231.33 \\
\hline 7 & Luteolin & 1.97 & 111.12 & 21 & 286.24 & 6 & 4 & 0 & 1 & 232.07 \\
\hline
\end{tabular}

Table 5

ADME studies of Uncaria tomentosa 


\begin{tabular}{|c|c|c|c|c|c|c|c|c|c|c|}
\hline SL. & Compounds & Mi & TSPA & Natoms & $\mathrm{mw}$ & no & nOHNH & $\mathbf{N}$ & nROTB & VOLUME \\
\hline NO & & $\log p$ & & & & $\mathbf{N}$ & & Violations & & \\
\hline 1 & Uncarine C & 2.18 & 67.88 & 27 & 368.43 & 6 & 1 & 0 & 2 & 333.36 \\
\hline 2 & Ajmalicine & 3.41 & 54.57 & 26 & 352.43 & 5 & 1 & 0 & 2 & 325.29 \\
\hline 3 & Isomitraphylline & 2.18 & 67.88 & 27 & 368.43 & 6 & 1 & 0 & 2 & 333.36 \\
\hline 4 & Isopteropodine & 2.18 & 67.88 & 27 & 368.43 & 6 & 1 & 0 & 2 & 333.36 \\
\hline 5 & Mitraphylline & 2.18 & 67.88 & 27 & 368.43 & 6 & 1 & 0 & 2 & 333.36 \\
\hline 6 & Isorhynchophylline & 2.83 & 67.88 & 28 & 384.48 & 6 & 1 & 0 & 5 & 360.74 \\
\hline 7 & Speciophylline & 2.18 & 67.88 & 27 & 368.43 & 6 & 1 & 0 & 2 & 333.36 \\
\hline
\end{tabular}

Table 6

ADME studies of Lycoris radiata 


\begin{tabular}{|c|c|c|c|c|c|c|c|c|c|c|}
\hline $\begin{array}{l}\text { SL. } \\
\text { NO }\end{array}$ & Compounds & $\begin{array}{l}\text { Mi } \\
\text { Log p }\end{array}$ & TSPA & Natoms & $\mathrm{mw}$ & nON & nOHNH & $\begin{array}{l}\mathbf{N} \\
\text { Violations }\end{array}$ & nROTB & VOLUME \\
\hline 1 & Caranine & 1.45 & 41.93 & 20 & 271.32 & 4 & 1 & 0 & 0 & 241.36 \\
\hline 2 & galanthamine & 1.54 & 41.93 & 21 & 287.36 & 4 & 1 & 0 & 1 & 268.19 \\
\hline 3 & Hippeastrine. & 1.23 & 68.24 & 23 & 315.32 & 6 & 1 & 0 & 0 & 269.11 \\
\hline 4 & Homolycorine & 1.90 & 48.01 & 23 & 315.37 & 5 & 0 & 0 & 2 & 288.23 \\
\hline 5 & Lycoramine & 1.56 & 41.93 & 21 & 289.38 & 4 & 1 & 0 & 1 & 274.38 \\
\hline 6 & lycorine & 0.54 & 62.16 & 21 & 287.31 & 5 & 2 & 0 & 0 & 249.40 \\
\hline 7 & Tazettine & -2.45 & 57.16 & 25 & 346.40 & 6 & 1 & 0 & 1 & 311.82 \\
\hline
\end{tabular}

Table 7

ADME studies of Glycyrrhiza glabra 


\begin{tabular}{|c|c|c|c|c|c|c|c|c|c|c|}
\hline $\begin{array}{l}\text { SL. } \\
\text { NO }\end{array}$ & Compounds & $\begin{array}{l}\text { Mi } \\
\text { Log } \\
\text { p }\end{array}$ & TSPA & Natoms & $\mathrm{mw}$ & nON & nOHNH & $\begin{array}{l}\mathrm{N} \\
\text { Violations }\end{array}$ & nROTB & VOLUME \\
\hline 1 & phloretin & 2.66 & 97.98 & 20 & 274.27 & 5 & 4 & 0 & 4 & 240.11 \\
\hline 2 & Rosmarinic acid & 1.63 & 144.52 & 26 & 360.32 & 8 & 5 & 0 & 7 & 303.54 \\
\hline 3 & Isocoumarin & 1.82 & 30.21 & 11 & 146.15 & 2 & 0 & 0 & 0 & 128.59 \\
\hline 4 & Cortison & 1.43 & 91.67 & 26 & 360.45 & 5 & 2 & 0 & 2 & 337.20 \\
\hline 5 & Liquiritigenin. & 2.20 & 66.76 & 19 & 256.26 & 4 & 2 & 0 & 1 & 222.24 \\
\hline 6 & Liquiritin & 0.41 & 145.91 & 30 & 418.40 & 9 & 5 & 0 & 4 & 354.37 \\
\hline 7 & Glabridin & 4.20 & 58.92 & 24 & 324.38 & 4 & 2 & 0 & 1 & 295.25 \\
\hline 8 & Chlorozotocin & -1.48 & 159.75 & 20 & 313.69 & 10 & 5 & 0 & 9 & 253.94 \\
\hline 9 & hispaglabridinA & 6.24 & 58.92 & 29 & 392.50 & 4 & 2 & 1 & 3 & 372.59 \\
\hline 10 & Isoliquiritin & 0.98 & 156.91 & 30 & 418.40 & 9 & 6 & 1 & 6 & 358.03 \\
\hline 11 & $\begin{array}{l}\text { O- } \\
\text { methylglabridin }\end{array}$ & 4.04 & 68.16 & 26 & 354.40 & 5 & 2 & 0 & 2 & 320.79 \\
\hline
\end{tabular}

It is seen that phytocompounds Ginkgolide C, hispaglabridinA and Isoliquiritin have 1 violations each in Lipinski's rule of 5 and hence can't be considered as ligands.

\section{Molecular Docking:}

Further docking is performed with the receptors in Table 4 with the above phytocompounds (Table 4-7). Docking scores, interacting amino acids along with number of interactions are noted in Table 8-11.

Table 8

Docking of CCR5 receptor 


\begin{tabular}{|c|c|c|c|c|}
\hline \multicolumn{5}{|l|}{ Plant: Ginkgo biloba } \\
\hline \multirow[t]{2}{*}{ COMPOUND } & $\begin{array}{l}\text { DOCKING } \\
\text { SCORE }\end{array}$ & INTERACTING AMINO ACID & \multirow[t]{2}{*}{ NO. OF INTERACTIONS } & \\
\hline & (Kcal/mol) & & & \\
\hline Ginkgolide A & -4.234 & THR-107, GLU-336 & 2 & \\
\hline Ginkgolide B & -4.342 & $\begin{array}{l}\text { ASN-311, THR- 312, THR-197, } \\
\text { TYR-304, }\end{array}$ & 5 & \\
\hline Ginkgolide C & -4.248 & $\begin{array}{l}\text { ASP-286, THR-67, ASP-68, ARG- } \\
128\end{array}$ & 7 & \\
\hline Isorhamnetin & -4.236 & THR-337, TYR-39, CYS-180 & 4 & \\
\hline Quercetin & -3.880 & THR-107 & 1 & \\
\hline $\begin{array}{l}\text { Protocatechuic } \\
\text { acid. }\end{array}$ & -3.840 & $\begin{array}{l}\text { ASP-68, ARG-128, ARG-142, THR- } \\
67, \text { SER-65 }\end{array}$ & 7 & \\
\hline Luteolin & -3.822 & ASP-68, GLU-355 & 3 & \\
\hline \multicolumn{5}{|c|}{ Plant:Uncaria tomentosa } \\
\hline Uncarine c & -4.742 & TRY-110, GLU-335 & 2 & \\
\hline Ajmalicine & -4.824 & GLU-336 & 1 & \\
\hline Isomitraphylline & -4.694 & GLU-336, THR-107 & 2 & \\
\hline Isopteropodine & -5.038 & ASP-286 & 1 & \\
\hline Mitraphylline & -4.610 & THR-107 & 1 & \\
\hline Isorhynchophylline & -5.204 & GLU-336 & 1 & \\
\hline Speciophylline & -4.586 & GLU-336, TYR-304 & 2 & \\
\hline \multicolumn{5}{|c|}{ Plant: Lycoris radiate } \\
\hline Caranine & & -3.740 & ARG-128, ARG-142 & 3 \\
\hline Tazettine & & -4.428 & TYR-110 & 1 \\
\hline Lycorine & & -3.952 & GLU-355 & 1 \\
\hline Lycoramine & & -4.178 & SER-65 & 1 \\
\hline Homolycorine & & -4.332 & SER-182 & 1 \\
\hline Hippeastrine & & -3.980 & ASP-286, LYS-64 & 2 \\
\hline galanthamine & & -4.042 & ASN-311 & 1 \\
\hline \multicolumn{5}{|c|}{ Plant:Glycyrrhiza glabra } \\
\hline phloretin & & -3.852 & THR-107 & 1 \\
\hline Rosmarinic acid & & -4.824 & $\begin{array}{l}\text { TYR-304, ASN-311, THR-312, } \\
\text { THR-197 }\end{array}$ & 5 \\
\hline Isocoumarin & & -2.728 & SER-65, THR-67 & 2 \\
\hline Cortison & & -4.368 & TYR-304, GLU-336, TYR-110 & 3 \\
\hline Liquiritigenin & & -3.666 & ASP-68, ARG-142 & 3 \\
\hline
\end{tabular}

Page 16/28 


\begin{tabular}{|llll|}
\hline Liquiritin & -4.958 & SER-65, ASP-68,GLU-355, & 4 \\
\hline Glabridin & & ARG-288 & 1 \\
\hline Chlorozotocin & -4.614 & GLU-355 & 3 \\
\hline hispaglabridinA & -4.074 & GLU-356,SER-65 & 2 \\
\hline Isoliquiritin & -5.512 & ASP-68, ARG-285 & 8 \\
& -5.302 & VAL-353,ARG-288, & \\
\hline O-methylglabridin & & ARG-285,ASP-68, SER-65,LEU- & 2 \\
\hline
\end{tabular}

Table 9

Docking of IFITM2 receptor 


\begin{tabular}{|c|c|c|c|}
\hline \multicolumn{4}{|l|}{ Plant: Ginkgo biloba } \\
\hline COMPOUND & $\begin{array}{l}\text { DOCKING SCORE } \\
\text { (Kcal/mol) }\end{array}$ & INTERACTING AMINO ACID & NO. OF INTERACTIONS \\
\hline Ginkgolide A & -4.556 & THR-215, VAL-145, VAL-211, VAL-131 & 4 \\
\hline Ginkgolide B & -4.546 & GLU-61 & 1 \\
\hline Ginkgolide C & -4.592 & VAL-131, THR-215 & 2 \\
\hline Isorhamnetin & -4.654 & VAL-145, THR-215 & 2 \\
\hline Quercetin & -4.372 & ALA-60 & 1 \\
\hline Protocatechuic acid & -4.660 & THR-195 & 1 \\
\hline Luteolin & -4.378 & VAL-145, ILE-150 & 3 \\
\hline \multicolumn{4}{|c|}{ Plant:Uncaria tomentosa } \\
\hline Uncarine C & -5.064 & VAL-131, ILE-127 & 2 \\
\hline Ajmalicine & -5.336 & ALA-16, THR-14, VAL-21, ASN-90 & 4 \\
\hline Isomitraphylline & -4.876 & GLU-58 & 1 \\
\hline Isopteropodine & -4.966 & VAL-211, THR-215 & 2 \\
\hline Mitraphylline & -4.686 & THR-215 & 1 \\
\hline Isorhynchophylline & -5.304 & ILE-127, VAL-211 & 2 \\
\hline Speciophylline & -4.832 & ILE-127 & 1 \\
\hline \multicolumn{4}{|l|}{ Plant: Lycoris radiate } \\
\hline Caranine & -4.498 & ILE-50 & 1 \\
\hline galanthamine & -4.754 & THR-14, THR-23 & 2 \\
\hline Hippeastrine. & -4.716 & ASP-90,TYR-85, HIS-57, ALA-60 & 5 \\
\hline Homolycorine & -4.780 & VAL -211 & 1 \\
\hline Lycoramine & -4.428 & ASN-90, TSR-14, GLY-12 & 3 \\
\hline lycorine & -4.668 & ILE-150 & 1 \\
\hline Tazettine & -4.620 & TYR-85, ASN-90 & 2 \\
\hline \multicolumn{4}{|c|}{ Plant:Glycyrrhiza glabra } \\
\hline Cortison & -4.972 & ILE-150, ALA-126, GLU-187 & 3 \\
\hline Glabridin & -5.038 & LYS-102 & 1 \\
\hline Hispaglabridin A & -5.848 & VAL-131 & 1 \\
\hline Isocoumarin & -2.986 & GLY-152 & 1 \\
\hline Isoliquiritin & -5.744 & THR-193, ILE-150, ASP-81, GLU-187 & 4 \\
\hline Liquiritigenin & -4.244 & ASN-97 & 1 \\
\hline Liquiritin & -5.582 & THR-198, ILE-88, GLY-146, VAL-145 & 3 \\
\hline
\end{tabular}




\begin{tabular}{|llll|} 
O-methylglabridin & -5.378 & GLY-152 & 1 \\
\hline phloretin & -4.852 & TYR-85, ASP-90 & 2 \\
\hline Rosmarinicacid & -5.508 & LYS-102, ASP-90 & 2 \\
\hline Chlorozotocin & -4.668 & TYR-85, SER-84, ASP-90 & 5 \\
\hline
\end{tabular}

Table 10

Docking of CLEC4M receptor 


\begin{tabular}{|c|c|c|c|}
\hline \multicolumn{4}{|l|}{ Plant: Ginkgo biloba } \\
\hline COMPOUND & $\begin{array}{l}\text { DOCKING SCORE } \\
\text { (Kcal/mol) }\end{array}$ & INTERACTING AMINO ACID & NO. OF INTERACTIONS \\
\hline Ginkgolide A & -3714 & GLN-87, ASN-63 & 2 \\
\hline Ginkgolide B & -3776 & GLN-87, GLN-10 & 3 \\
\hline Ginkgolide C & -3686 & GLN-87, LYS-175 & 3 \\
\hline Isorhamnetin & -3420 & ARG-51 & 1 \\
\hline Quercetin & -3422 & THR-40, THR-17, THR-13, LYS-43 & 4 \\
\hline Protocatechuic acid & -3076 & THR-40, LYS-20 & 2 \\
\hline Luteolin & -3330 & LYS-176 & 1 \\
\hline \multicolumn{4}{|c|}{ Plant:Uncaria tomentosa } \\
\hline Uncarine C & -4182 & ASN-63 & 1 \\
\hline Ajmalicine & -4178 & GLN-87 & 1 \\
\hline Isomitraphylline & -4390 & THR-58, ASP-56 & 1 \\
\hline Isopteropodine & -4178 & GLN-87 & 1 \\
\hline Mitraphylline & -4086 & GLM-84 & 1 \\
\hline Isorhynchophylline & -4332 & ASN-63 & 1 \\
\hline Speciophylline & -3936 & GLN-10,LYS-175, ASN-63 & 4 \\
\hline \multicolumn{4}{|l|}{ Plant: Lycoris radiate } \\
\hline Caranine & -3340 & ASN-69,MET-67. & 2 \\
\hline galanthamine & -3602 & THR-17 & 1 \\
\hline Hippeastrine. & -3484 & LYS-175 & 1 \\
\hline Homolycorine & -3758 & LYS-20 & 1 \\
\hline Lycoramine & -3662 & LYS-20, THR 40 & 2 \\
\hline lycorine & -3458 & MET-67, THR-58 & 2 \\
\hline Tazettine & -3770 & LYS-92 & 1 \\
\hline \multicolumn{4}{|c|}{ Plant:Glycyrrhiza glabra } \\
\hline Cortison & -3714 & ASN-63, GLN-87, TYR-13 & 3 \\
\hline Glabridin & -3906 & LYS-176, GLU-5, LYS-8 & 3 \\
\hline HispaglabridinA & -4884 & ASN-63 & 1 \\
\hline Isocoumarin & -2296 & THR-40 & 1 \\
\hline Isoliquiritin & -4250 & CYS-180, ARG-182 & 2 \\
\hline Liquiritigenin & -3310 & LYS-8,LYS-176,PHE-181,ALA-178 & 4 \\
\hline Liquiritin & -4408 & GLN-87, ARG-85 & 2 \\
\hline
\end{tabular}

Page 20/28 


\begin{tabular}{|llll|} 
O-methylglabridin & -4268 & ARG-109, THR-58 & 2 \\
\hline phloretin & -3390 & GLU-5, ARG-85,VAL-84 & 3 \\
\hline Rosmarinicacid & -4488 & LYS-176 & 1 \\
\hline Chlorozotocin & -3532 & LYS-176, GLU-5, LYS-8 & 3 \\
\hline
\end{tabular}

Table 11

Docking of IFR3 receptor 


\begin{tabular}{|c|c|c|c|}
\hline \multicolumn{4}{|l|}{ Plant: Ginkgo biloba } \\
\hline $\begin{array}{l}\text { NO. OF } \\
\text { INTERACTIONS }\end{array}$ & $\begin{array}{l}\text { NO. OF } \\
\text { INTERACTIONS }\end{array}$ & NO. OF INTERACTIONS & $\begin{array}{l}\text { NO. OF } \\
\text { INTERACTIONS }\end{array}$ \\
\hline Ginkgolide A & -4966 & PRO-153, SER-152, HIS-151, ARG-25, ARG-193 & 6 \\
\hline Ginkgolide B & -5054 & $\begin{array}{l}\text { SER-152, GLU-110, ARG-193, SER-152, GLY-26, } \\
\text { ARG-25 }\end{array}$ & 6 \\
\hline Ginkgolide C & -4842 & TYR-155, GLU-111, GLY-26, VAL-172, ARG-25, & 6 \\
\hline Isorhamnetin & -4672 & ARG-25, ARG-27, SER-152, LEU-200 & 4 \\
\hline Quercetin & -4388 & $\begin{array}{l}\text { LEU-200, ARG-193, ARG-27, ARG-25, TYR-155, } \\
\text { THR-156 }\end{array}$ & 6 \\
\hline Protocatechuic acid & -4052 & GLY-26, TYR-155, SER-152 ARG-193 & 4 \\
\hline Luteolin & -4300 & ARG-27, LYS-173, GLU-111, LEU-200, ARG-25 & 6 \\
\hline \multicolumn{4}{|c|}{ Plant: Uncaria tomentosa } \\
\hline Uncarine c & -5362 & GLU-110, SER-152, LEU-200, LYS-173, ARG-25 & 5 \\
\hline Ajmalicine & -5208 & GLU-111, SER-152, GLU-110, TYR-155 & 4 \\
\hline Isomitraphylline & -5246 & SER-152, GLU-110, TYR-155, ARG-25 & 4 \\
\hline Isopteropodine & -4892 & ARG-25, ARG-193, GLU-110 & 3 \\
\hline Mitraphylline & -5292 & LYS-152, SER-152 & 2 \\
\hline Isorhynchophylline & -5338 & ARG-25, GLU-110,TYR-155, ARG-193 & 5 \\
\hline Speciophylline & -5068 & LEU-200,GLU-110, ARG-25 & 3 \\
\hline \multicolumn{4}{|l|}{ Plant: Lycoris radiate } \\
\hline Caranine & -4180 & ARG-25 & 1 \\
\hline galanthamine & -4632 & LYS-173, ARG-25 & 2 \\
\hline Hippeastrine. & -4644 & SER-152 & 1 \\
\hline Homolycorine & -5034 & SER-152, GLU-110,LYS-173, & 1 \\
\hline Lycoramine & -4508 & LEU-200, GLU 110 & 2 \\
\hline lycorine & -4418 & SER-152, VAL-172, ARG-25, GLU-110 & 4 \\
\hline Tazettine & -5380 & SER-152, ARG-25 & 2 \\
\hline \multicolumn{4}{|c|}{ Plant: Glycyrrhiza glabra } \\
\hline Cortison & -5186 & GLY-150, TYR-155, ARG-25, ARG-27, GLU-111 & 6 \\
\hline Glabridin & -5092 & ARG-193, SER-152, LEU-200, ARG-25 & 2 \\
\hline Hispaglabridin A & -6154 & ARG-25 & 1 \\
\hline Isocoumarin & -2978 & LYS-173, GLU-110 & 2 \\
\hline Isoliquiritin & -5440 & ARG-154,ARG-27, ARG-193, TYR-155 & 4 \\
\hline Liquiritigenin & -4388 & GLY-26, LYS-173, GLY-150,LEU-200, TYR-155 & 5 \\
\hline Liquiritin & -5706 & HIS-151, GLU-110, TYR-155, ARG-25, & 6 \\
\hline
\end{tabular}




\begin{tabular}{|llll|}
\hline O-methylglabridin & -5594 & TYR-155, ARG-193 & 2 \\
\hline phloretin & -4576 & $\begin{array}{l}\text { LEU-200, GLU-111, ARG-193, ASP-67, ARF-25, } \\
\text { SER-152 }\end{array}$ & 5 \\
\hline Rosmarinicacid & -5214 & $\begin{array}{l}\text { ASP-67, ARG-193, SER-198, LEU-200, LYS-173, } \\
\text { GLU-111 }\end{array}$ & 6 \\
\hline Chlorozotocin & -4648 & ARG-25, TYR-155, SER-152, ASP-67 & 4 \\
\hline
\end{tabular}

As per docking studies it is seen that phytocompounds Ginkgolide A, Ginkgolide B, Luteolin and Protocatechuic acid from Ginkgo biloba and Liquiritin, Cortison and Chlorozotocin from Glycyrrhiza glabra have good interactions with the West Nile fever gene receptors.

\section{Conclusion}

The taxonomy and functional information of West Nile fever microbiome are identified. The Human abundance table and superfamily database identified superfamilies as P-loop containing nucleoside triphosphate hydrolases, Viral glycoprotein Family, DNA/RNA polymerases Family, E set domains, S-adenosyl-L-methionine-dependent methyltransferases,

Trypsin-like serine proteases, Flavivirus capsid protein C and S-adenosyl-L-methionine-dependent methyltransferases and of the families RNA helicase, Viral glycoprotein, Class II viral fusion proteins C-terminal domain, RNA-dependent RNApolymerase, mRNA cap methylase, Viral proteases, Flavivirus capsid protein C and mRNA cap methylase.

Again, as per docking studies and ADME analysis it is seen that phytocompounds Ginkgolide A, Ginkgolide B, Luteolin and Protocatechuic acid from Ginkgo biloba can be potential ligands for the receptors implicated in West Nile Fever. Again, phytocompounds Liquiritin, Cortison and Chlorozotocin from Glycyrrhiza glabra can be potential ligands for the receptors implicated in West Nile Fever as per ADME analysis and docking studies. Further, in-vitro and in-vivo studies can be done on the above phytocompounds to establish their potential as drugs in treating West Nile Fever.

\section{Declarations}

1. Ethics approval and consent to participate: Not applicable

2. Consent for publication: Yes

3. Availability of data and materials: 


\begin{tabular}{|lll|}
\hline $\begin{array}{l}\text { Item. } \\
\text { No. }\end{array}$ & Data & Resource \\
\hline 1. & $\begin{array}{l}\text { Microbiome with accession number } \\
\text { SRR10017187 }\end{array}$ & $\begin{array}{l}\text { https://www.ncbi.nlm.nih.gov/sra/? } \\
\text { term=SRR10017187 }\end{array}$ \\
\hline 2. & CCR5 with accession number AAB57793.1 & https://www.ncbi.nlm.nih.gov/protein/AAB57793.1 \\
\hline 3. & CLEC4M with accession number AAl10615.1 & https://www.ncbi.nlm.nih.gov/protein/AAI10615.1 \\
\hline 4. & IFITM2 with accession number CAG33175.1 & https://www.ncbi.nlm.nih.gov/protein/CAG33175.1 \\
\hline 5. & IRF3 with accession number AAH71721.1 & https://www.ncbi.nlm.nih.gov/protein/AAH71721.1 \\
\hline 6. & 4MBS & https://www.rcsb.org/structure/4MBS \\
\hline 7. & 1SL6 & https://www.rcsb.org/structure/1SL6 \\
\hline 8. & 3WXX & https://www.rcsb.org/structure/3WXX \\
\hline 9. & $7 J F M$ & https://www.rcsb.org/structure/7JFM \\
\hline
\end{tabular}

4. Competing interests: None

5. Funding: None

6. Authors' contributions: All authors have equal contribution

7. Acknowledgements: We would like to thank our institution for providing lab support

\section{References}

1. Duguma, D., Hall, M. W., Smartt, C. T., Debboun, M., \& Neufeld, J. D. (2019). Microbiota variations in Culex nigripalpus disease vector mosquito of West Nile virus and Saint Louis Encephalitis from different geographic origins. PeerJ, 6, e6168.

2. Ezgimen, M., Lai, H., Mueller, N. H., Lee, K., Cuny, G., Ostrov, D. A., \& Padmanabhan, R. (2012). Characterization of the 8hydroxyquinoline scaffold for inhibitors of West Nile virus serine protease. Antiviral research, 94(1), 18-24.

3. Petersen, L. R., Brault, A. C., \&Nasci, R. S. (2013). West Nile virus: review of the literature. Jama, 310(3), 308-315.

4. Kramer, L. D., Li, J., \& Shi, P. Y. (2007). West nile virus. The Lancet Neurology, 6(2), 171-181.

5. Blankenberg, D., \& Hillman-Jackson, J. (2014). Analysis of next-generation sequencing data using Galaxy. In Stem cell transcriptional networks (pp. 21-43). Humana Press, New York, NY.

6. Bashiardes S, Zilberman-Schapira G, and Elinav E, (2016), Use of Metatranscriptomics in Microbiome Research, Bioinform Biol Insights. ; 10: 19-25.

7. Handelsman J, (2004), Metagenomics: Application of Genomics to Uncultured Microorganisms, Microbiol Mol Biol Rev. 68(4): 669-685.

8. Waterhouse A, Bertoni M, Bienert S, Studer G, Tauriello G, Gumienny R, Heer FT, A P de Beer T, Rempfer C, Bordoli L, Lepore R and Schwede T, (2018), SWISS-MODEL: homology modelling of protein structures and complexes, Nucleic Acids Res.; 46(Web Server issue): W296-W303.

9. Vyas VK, Ukawala RD, Ghate M, and Chintha C (2012), Homology Modeling a Fast Tool for Drug Discovery: Current Perspectives, Indian J Pharm Sci.74(1): 1-17.

10. Morris GM, Lim-Wilby M, (2008), Molecular docking, Methods Mol Biol, 443:365-82.

11. Andrews, S. (n.d.). FastQC A Quality Control tool for High Throughput Sequence Data. Retrieved from http://www.bioinformatics.babraham.ac.uk/projects/fastqc/ 
12. Ewels, P., Magnusson, M., Lundin, S., \&KÃaller, M. (2016). MultiQC: summarize analysis results for multiple tools and samples in a single report. Bioinformatics, 32(19), 3047-3048. https://doi.org/10.1093/bioinformatics/btw354

13. Kopylova, E., Noé, L., \&Touzet, H. (2012). SortMeRNA: fast and accurate filtering of ribosomal RNAs in metatranscriptomic data. Bioinformatics, 28(24), 3211-3217. https://doi.org/10.1093/bioinformatics/bts611

14. Quast, C., Pruesse, E., Yilmaz, P., Gerken, J., Schweer, T., Yarza, P., ... Glöckner, F. O. (2012). The SILVA ribosomal RNA gene database project: improved data processing and web-based tools. Nucleic Acids Research, 41(D1), D590-D596. https://doi.org/10.1093/nar/gks1219

15. Burge, S. W., Daub, J., Eberhardt, R., Tate, J., Barquist, L., Nawrocki, E. P., ... Bateman, A. (2012). Rfam 11.0: 10 years of RNA families. Nucleic Acids Research, 41(D1), D226-D232. https://doi.org/10.1093/nar/gks1005

16. Edgar, R. C. (2010). Search and clustering orders of magnitude faster than BLAST. Bioinformatics, 26(19), 2460-2461. https://doi.org/10.1093/bioinformatics/btq461

17. Loman, N. J., Misra, R. V., Dallman, T. J., Constantinidou, C., Gharbia, S. E., Wain, J., \&Pallen, M. J. (2012). Performance comparison of benchtop high-throughput sequencing platforms. Nature Biotechnology, 30(5), 434-439. https://doi.org/10.1038/nbt.2198

18. Blankenberg, D., Gordon, A., Von Kuster, G., Coraor, N., Taylor, J., \& and, A. N. (2010). Manipulation of FASTQ data with Galaxy. Bioinformatics, 26(14), 1783-1785. https://doi.org/10.1093/bioinformatics/btq281

19. Beghini, F., Mclver, L. J., Blanco-Míguez, A., Dubois, L., Asnicar, F., Maharjan, S., ... Segata, N. (2021). Integrating taxonomic, functional, and strain-level profiling of diverse microbial communities with bioBakery 3. ELife, 10. https://doi.org/10.7554/elife.65088

20. Ondov, B. D., Bergman, N. H., \&Phillippy, A. M. (2011). Interactive metagenomic visualization in a Web browser. BMC Bioinformatics, 12(1). https://doi.org/10.1186/1471-2105-12-385

21. Cuccuru, G., Orsini, M., Pinna, A., Sbardellati, A., Soranzo, N., Travaglione, A., ... Fotia, G. (2014). Orione, a web-based framework for NGS analysis in microbiology. Bioinformatics, 30(13), 1928-1929.

https://doi.org/10.1093/bioinformatics/btu135

22. Asnicar, F., Weingart, G., Tickle, T. L., Huttenhower, C., \&Segata, N. (2015). Compact graphical representation of phylogenetic data and metadata with GraPhIAn. PeerJ, 3, e1029. https://doi.org/10.7717/peerj.1029

23. Abubucker, S., Segata, N., Goll, J., Schubert, A. M., Izard, J., Cantarel, B. L., ... Huttenhower, C. (2012). Metabolic Reconstruction for Metagenomic Data and Its Application to the Human Microbiome. PLoS Computational Biology, 8(6), e1002358. https://doi.org/10.1371/journal.pcbi.1002358

24. https://www.molinspiration.com, SlovenskyGrob, Slovakia

25. Duhovny D, Nussinov R, Wolfson HJ. Efficient Unbound Docking of Rigid Molecules. In Gusfield et al., Ed. Proceedings of the 2'nd Workshop on Algorithms in Bioinformatics (WABI) Rome, Italy, Lecture Notes in Computer Science 2452, pp. 185-200, Springer Verlag, 2002

26. Schneidman-Duhovny D, Inbar Y, Nussinov R, Wolfson HJ. PatchDock and SymmDock: servers for rigid and symmetric docking. Nucl. Acids. Res. 33: W363-367, 2005.

27. Martin, M. (2011). Cutadapt removes adapter sequences from high-throughput sequencing reads. EMBnet.Journal, 17(1), 10. https://doi.org/10.14806/ej.17.1.200

28. Jagtap P, Mehta S, Sajulga R, Batut B, Leith E, Kumar P, Hiltemann S, 2021 Metatranscriptomics analysis using microbiome RNA-seq data (Galaxy Training Materials).

29. Gough, J., Karplus, K., Hughey, R. and Chothia, C. (2001). Assignment of Homology to Genome Sequences using a Library of Hidden Markov Models that Represent all Proteins of Known Structure. J. Mol. Biol., 313(4), 903-919

\section{Figures}




$$
0
$$

Figure 1

Generation, personalization and annotation of tree: Tree in PhyloXML

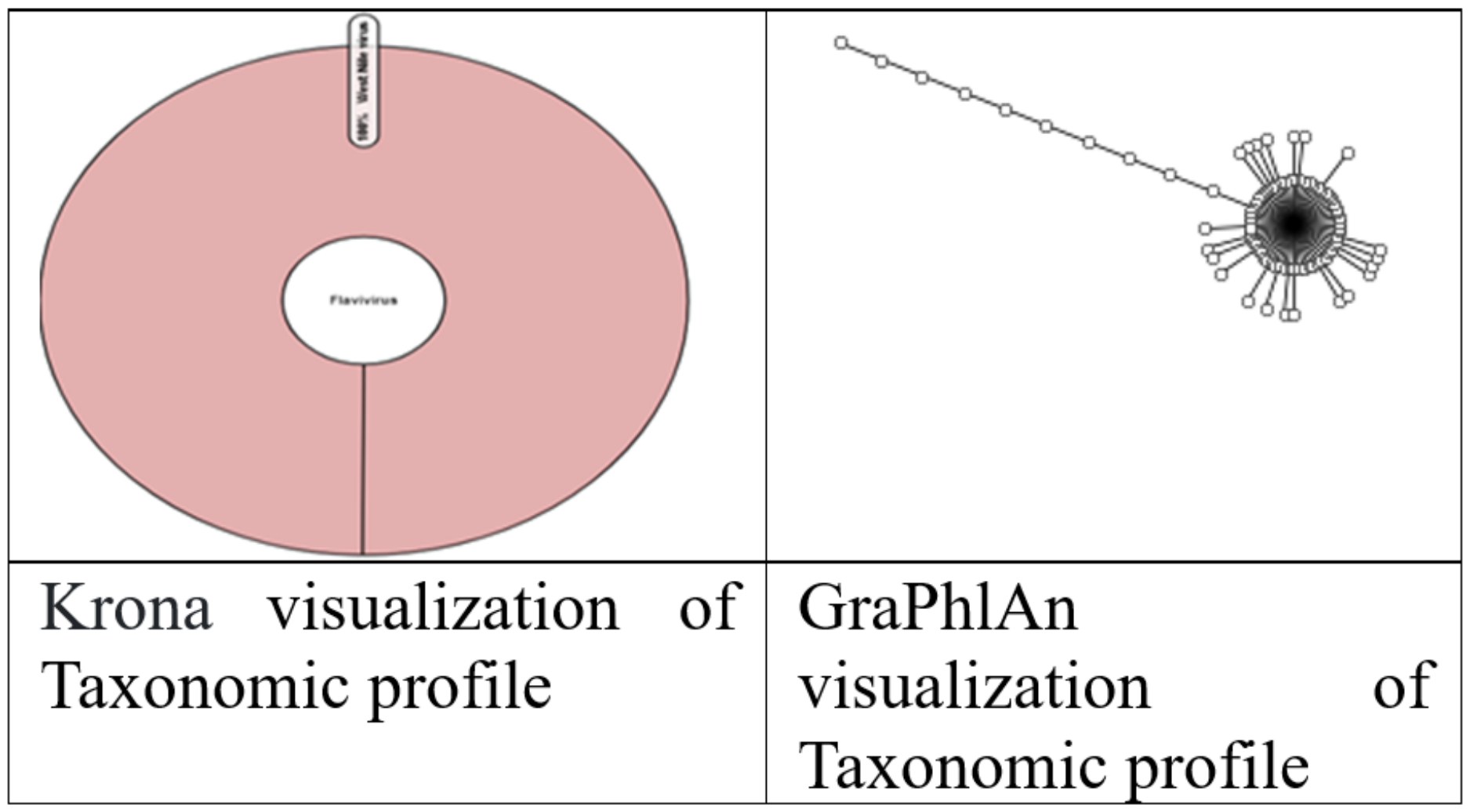

Figure 2

Visualization of Taxonomic profile in Krona and GraPhIAn 


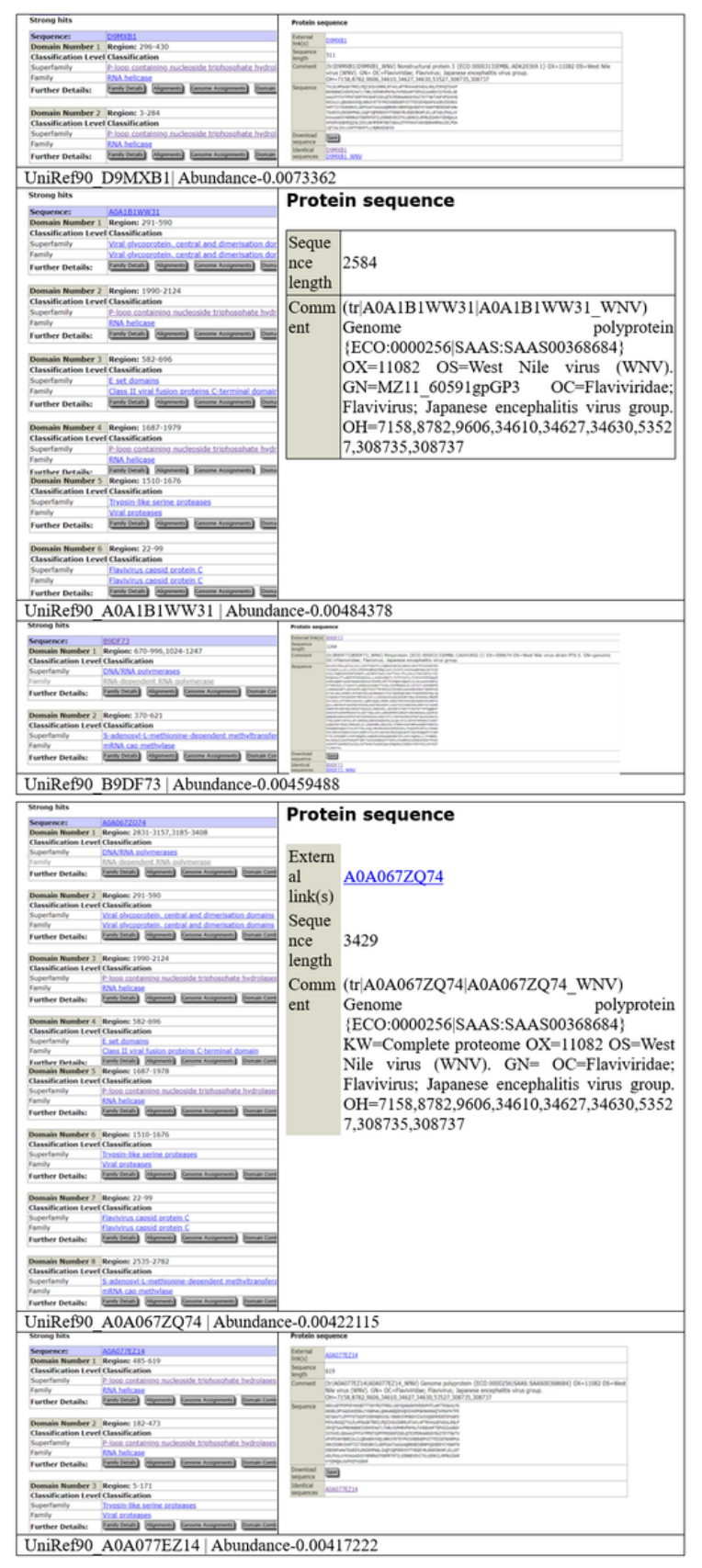

\section{Figure 3}

Functional information of $1^{\text {st }}$ five gene families as detected by Superfamily server [29] 

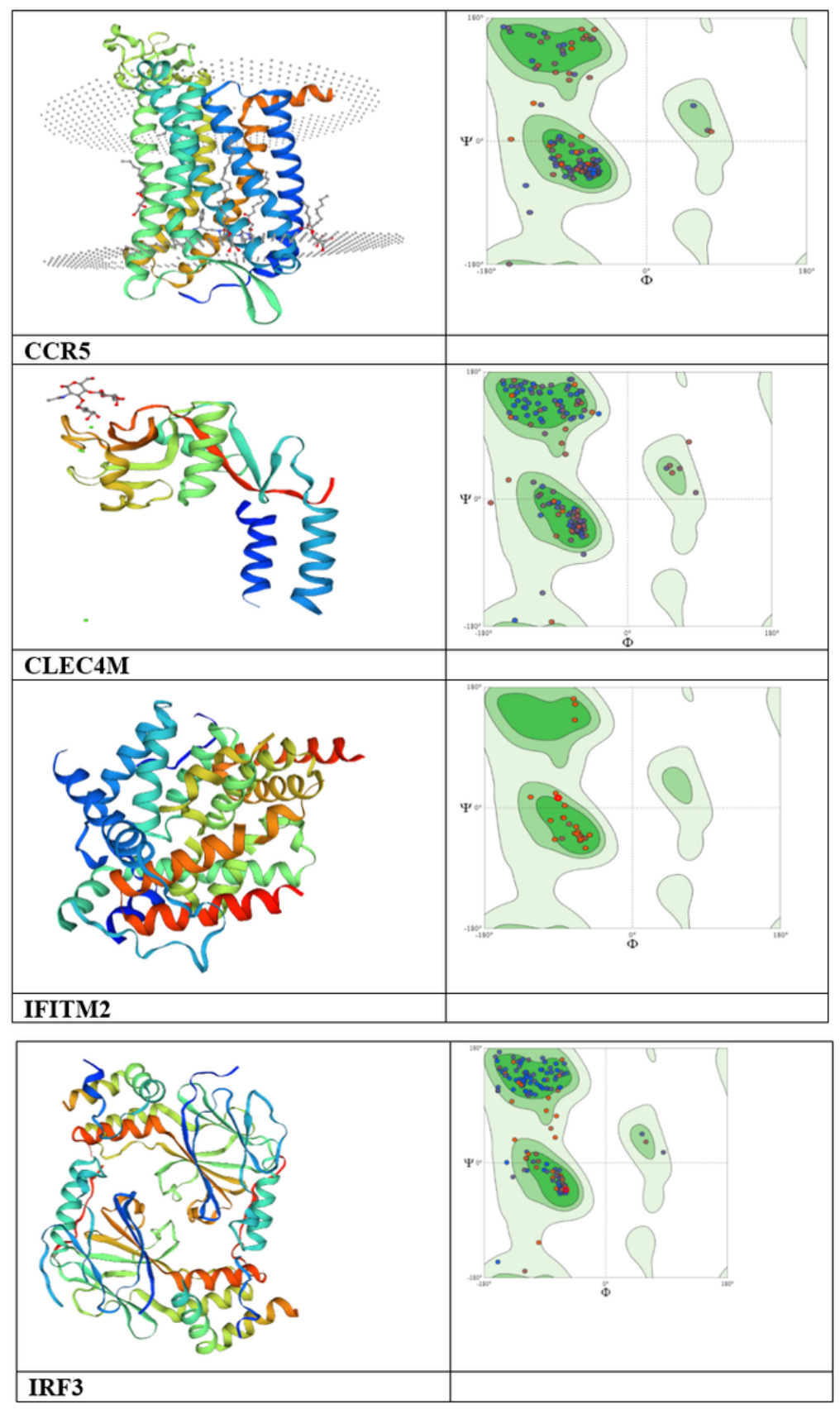

Figure 4

Swiss-model generated receptor models with their ramachandran plot 